\section{High prognostic value of measurable residual disease detection by flow cytometry in chronic lymphocytic leukemia patients treated with front-line fludarabine, cyclophosphamide, and rituximab, followed by three years of rituximab maintenance}

José A. García-Marco, ${ }^{1}$ Javier López Jiménez, ${ }^{2}$ Valle Recasens, ${ }^{3}$ Miguel Fernández Zarzoso, ${ }^{4}$ Eva González-Barca, ${ }^{5}$ Nieves Somolinos De Marcos, ${ }^{6}$ M. Jose Ramírez, ${ }^{7}$ Francisco Javier Peñalver Parraga, ${ }^{8}$ Lucrecia Yañez, ${ }^{9}$ Javier De La Serna Torroba, ${ }^{10}$ Maria Dolores Garcia Malo, ${ }^{11}$ Guillermo Deben Ariznavarreta, ${ }^{12}$ Ernesto Perez Persona, ${ }^{13}$ M. Angeles Ruiz Guinaldo, ${ }^{14}$ Raquel De Paz Arias, ${ }^{15}$ Elena Bañas Llanos, ${ }^{16}$ Isidro Jarque,${ }^{17}$ M. del Carmen Fernandez Valle ${ }^{18}$ Ana Carral Tatay, ${ }^{19}$ Jaime Perez De Oteyza, ${ }^{20}$ Eva Maria Donato Martin, ${ }^{21}$ Inmaculada Perez Fernández, ${ }^{22}$ Rafael Martinez Martinez, ${ }^{23}$ M. Angeles Andreu Costa, ${ }^{24}$ Diana Champ, ${ }^{25}$ Julio García Suarez, ${ }^{26}$ Marcos González Díaz, ${ }^{27}$ Secundino Ferrer, ${ }^{4}$ Félix Carbonell ${ }^{28}$ and José A. García-Vela ${ }^{29}$ on behalf of the GELLC Study Group

${ }^{1}$ Hematology, Hospital Universitario Puerta de Hierro-Majadahonda, Madrid; ${ }^{2}$ Hematology, Hospital Ramon y Cajal, Madrid; ${ }^{3}$ Hematology, Hospital Miguel Servet, Zaragoza; ${ }^{4}$ Hematology, Hospital Universitario Dr Peset, Valencia; ${ }^{5}$ Hematology, Institut Català d'Oncologia, L'Hospitalet de Llobregat, Barcelona; ${ }^{6} \mathrm{Hematology,} \mathrm{Hospital} \mathrm{Universitario} \mathrm{de}$ Getafe, Madrid; ${ }^{7}$ Hematology, Hospital de Jerez de la Frontera, Jerez; ${ }^{8}$ Fundación Hospital de Alcorcón, Madrid; ${ }^{9}$ Hospital Universitario Marqués de Valdecilla, Servicio de Hematologia, Santander; ${ }^{10}$ Department of Hematology, Hospital Doce de Octubre, Madrid; ${ }^{11} \mathrm{Hematology,} \mathrm{Hospital} \mathrm{Morales} \mathrm{Meseguer,} \mathrm{Murcia;}{ }^{12} \mathrm{Hematology,} \mathrm{CHU} \mathrm{Juan} \mathrm{Canalejo,} \mathrm{A}$ Coruña; ${ }^{13} \mathrm{Hematology}$, Hospital Txagorritxu, Vitoria; ${ }^{14} \mathrm{Hematology,} \mathrm{Hospital} \mathrm{Francesc} \mathrm{Borja,}$ Valencia; ${ }^{15} \mathrm{Hematology,} \mathrm{Hospital} \mathrm{La} \mathrm{Paz,} \mathrm{Madrid;}{ }^{16} \mathrm{Hematology,} \mathrm{Hospital} \mathrm{San} \mathrm{Pedro} \mathrm{de}$ Alcantara, Caceres; ${ }^{17} \mathrm{Hematology,} \mathrm{Hospital} \mathrm{Universitario} \mathrm{La} \mathrm{Fe,} \mathrm{Valencia;}{ }^{18} \mathrm{Hematology,}$ Hospital Universitario Puerta del Mar, Cadiz; ${ }^{19} \mathrm{Hematology,} \mathrm{Hospital} \mathrm{de} \mathrm{Sagunto,} \mathrm{Valencia;}$ ${ }^{20} \mathrm{Hematology,} \mathrm{Hospital} \mathrm{Madrid} \mathrm{Norte} \mathrm{Sanchinarro,} \mathrm{Madrid;}{ }^{21} \mathrm{Hematology,} \mathrm{Hospital} \mathrm{General}$ Castellon, Castellon; ${ }^{22}$ Hematology, Hospital Regional Universitario de Málaga, Málaga; ${ }^{23} \mathrm{Hematology,} \mathrm{Hospital} \mathrm{Clínico} \mathrm{Universitario} \mathrm{San} \mathrm{Carlos,} \mathrm{Madrid;}{ }^{24} \mathrm{Hematology,} \mathrm{Hospital}$ General de Móstoles, Madrid; ${ }^{25}$ Roche Farma, S.A., Madrid; ${ }^{26} \mathrm{Hematology,} \mathrm{Hospital}$ Universitario Príncipe Asturias, Madrid; ${ }^{27} \mathrm{Hematology,} \mathrm{University} \mathrm{Hospital} \mathrm{of} \mathrm{Salamanca-}$ IBSAL, CIBERONC, USAL-CSIC, CIC-IBMCC, Salamanca; ${ }^{28} \mathrm{Hematology,} \mathrm{Consorcio} \mathrm{Hospital}$ General Universitario, Valencia and ${ }^{29} \mathrm{Hematology,} \mathrm{Hospital} \mathrm{Universitario} \mathrm{de} \mathrm{Getafe,}$ Madrid, Spain

\section{ABSTRACT}

$\tau$ has been postulated that monitoring measurable residual disease (MRD) could be used as a surrogate marker of progression-free survival (PFS) in chronic lymphocytic leukemia (CLL) patients after treatment with immunochemotherapy regimens. In this study, we analyzed the outcome of 84 patients at 3 years of follow-up after first-line treatment with fludarabine, cyclophosphamide and rituximab (FCR) induction followed by 36 months of rituximab maintenance thearpy. MRD was assessed by a quantitative four-color flow cytometry panel with a sensitivity level of $10^{-4}$. Eighty out of 84 evaluable patients $(95.2 \%)$ achieved at least a partial response or better at the end of induction. After clinical evaluation, 74 patients went into rituximab maintenance and the primary endpoint was assessed in the final analysis at 3 years of follow-up. Bone marrow (BM) $\mathrm{MRD}$ analysis was performed after the last planned induction course and every 6 months in cases with detectable residual disease during the 36 months of maintenance therapy. Thirty-seven patients (44\%) did not have detectable residual disease in the BM prior to maintenance therapy. Interestingly, 29 patients with detectable residual disease in the BM after
Ferrata Storti Foundation

Haematologica 2019

Volume 104(11):2249-2257

\section{Correspondence:}

JOSÉ GARCÍA-MARCO

jagarciam@aehh.org

Received: August 20, 2018.

Accepted: March 18, 2019.

Pre-published: March 19, 2019.

doi:10.3324/haematol.2018.204891

Check the online version for the most updated information on this article, online supplements, and information on authorship \& disclosures: www.haematologica.org/content/104/11/2249

\section{(C)2019 Ferrata Storti Foundation}

Material published in Haematologica is covered by copyright. All rights are reserved to the Ferrata Storti Foundation. Use of published material is allowed under the following terms and conditions:

https://creativecommons.org/licenses/by-nc/4.0/legalcode. Copies of published material are allowed for personal or internal use. Sharing published material for non-commercial purposes is subject to the following conditions: https://creativecommons. org/licenses/by-nc/4.0/legalcode, sect. 3. Reproducing and sharing published material for commercial purposes is not allowed without permission in writing from the publisher. 
induction no longer had detectable disease in the BM following maintenance therapy. After a median followup of 6.30 years, the median overall survival (OS) and PFS had not been reached in patients with either undetectable or detectable residual disease in the BM, who had achieved a complete response at the time of starting maintenance therapy. Interestingly, univariate analysis showed that after rituximab maintenance OS was not affected by IGHV status (mutated $v$ s. unmutated OS: $85.7 \%$ alive at 7.2 years $v$ s. $79.6 \%$ alive at 7.3 years, respectively). As per protocol, 15 patients (17.8\%), who achieved a complete response and undetectable peripheral blood and BM residual disease after four courses of induction, were allowed to stop fludarabine and cyclophosphamide and complete two additional courses of rituximab and continue with maintenance therapy for 18 cycles. Surprisingly, the outcome in this population was similar to that observed in patients who received the full six cycles of the induction regimen. These data show that, compared to historic controls, patients treated with FCR followed by rituximab maintenance have high-quality responses with fewer relapses and improved OS. The tolerability of this regime is favorable. Furthermore, attaining an early undetectable residual disease status could shorten the duration of chemoimmunotherapy, reducing toxicities and preventing long-term side effects. The analysis of BM MRD after fludarabine-based induction could be a powerful predictor of post-maintenance outcomes in patients with CLL undergoing rituximab maintenance and could be a valuable tool to identify patients at high risk of relapse, influencing further treatment strategies. This trial is registered with EudraCT n. 2007-002733-36 and ClinicalTrials.gov Identifier: NCT00545714.

\section{Introduction}

Chronic lymphocytic leukemia (CLL) is a mature B-cell neoplasm characterized by a clonal proliferation and compartmentalized accumulation of neoplastic B cells within the blood, bone marrow and secondary lymphatic organs. The neoplastic B cells typically co-express CD5, and CD19, CD20, and CD23; compared with normal B cells, the levels of CD20 and CD79b on CLL cells are usually diminished. ${ }^{1-4}$ Mutations of immunoglobulin heavy variable chain (IGHV) genes and chromosomal abnormalities are the most important predictors of disease course.

For physically fit patients requiring treatment according to the International Workshop on CLL criteria, the combination of fludarabine, cyclophosphamide and the chimeric anti-CD20 antibody rituximab (FCR) is the standard of care for first-line treatment, based on the improvement of progression-free survival (PFS) and overall survival (OS) of patients treated with this combination compared with those treated with combination chemotherapy alone. ${ }^{5}$ Following the introduction of purine analogs as a treatment option, higher response rates and a higher proportion of complete remissions were observed, and even better outcomes have been reported in patients carrying mutated IGHV genes (excluding 11q or 17p deletions); patients treated with combination therapies such as FCR may achieve a life expectancy close to that observed in the matched normal general population. ${ }^{6-8}$

Achieving higher $C R$ rates with chemoimmunotherapy has translated into a documented increase in PFS and seems to lead to an OS benefit, as shown in the CLL8 trial, which reported a $33 \%$ reduced risk of death $(P=0.01)$ with the FCR regimen when compared to fludarabine plus cyclophosphamide as first-line therapy. However, while attainment of a CR has historically been considered the gold-standard for treatment response, many of these patients have persistent disease that cannot be easily identified by routine testing approaches. ${ }^{9}$ This, coupled with the development of extremely sensitive testing technologies, has led to the emergence of measurable residual disease (MRD) as an important endpoint in the treatment of
CLL, especially in the era of chemoimmunotherapy. Indeed, achieving undetectable MRD after chemoimmunotherapy is a desirable goal, as MRD below a threshold of $10^{-4}(0.01 \%)$ results in improvement of PFS and OS. We hypothesized that using MRD as a surrogate of treatment effectiveness would allow determination of the efficacy of new treatments without the need for prolonged observation.

Several studies have shown that sequential use of induction/maintenance treatment can improve the quality of response achieved with induction. Abrisqueta et al. recently reported an analysis of whether maintenance therapy can improve the response achieved with induction chemotherapy. ${ }^{10}$ Sixty-seven patients responding to induction therapy with FCR plus mitoxantrone (R-FCM) received rituximab maintenance therapy $\left(375 \mathrm{mg} / \mathrm{m}^{2}\right)$ every 3 months for 2 years. Approximately $40.6 \%$ of patients achieved a CR with undetectable MRD at the end of the maintenance treatment. It is important to note that $21 \%$ of the patients who had detectable MRD at the end of R-FCM induction had an improved response after rituximab maintenance therapy. Another study showed that after responding to a fludarabine induction, patients who had detectable MRD and were consolidated with four monthly cycles of rituximab followed by a maintenance regimen of 12 monthly rituximab doses had significantly longer responses, compared to those who did not receive consolidation (5-year OS: $87 \%$ vs. $32 \% ; P<0.001$ ). The estimated 5 -year PFS after induction was $73 \% .^{11}$

However, despite the improvements achieved with rituximab maintenance therapy, there are some biological features which confer a poor response to consolidation plus maintenance therapy. Dal Bo et al. showed that patients harboring the NOTCH1 mutation had a significantly shorter OS compared with those with unmutated NOTCH1. The independent prognostic impact of NOTCH1 mutation on OS was confirmed in multivariate analysis. ${ }^{12}$

In the light of these observations, we conducted a multicenter, non-randomized phase II clinical trial that aimed to evaluate the efficacy, in terms of CR rate, of FCR as 
first-line treatment for CLL, and to investigate the impact of rituximab maintenance therapy on the response rate and PFS following FCR. A key secondary objective was to analyze MRD status after chemoimmunotherapy and rituximab maintenance.

\section{Methods}

Physically fit patients between 18 and 70 years old with active $\mathrm{CD} 20^{+}$CLL according to the World Health Organization classification, with an Eastern Cooperative Oncology Group Performance Status $\leq 2$, were recruited into the REM (rituximab in maintenance) trial and received treatment with fludarabine (25 $\mathrm{mg} / \mathrm{m}^{2}$ iv on days $\left.1-3\right)$, cyclophosphamide $\left(250 \mathrm{mg} / \mathrm{m}^{2}\right.$ iv on days 1-3) and rituximab (375 mg/m iv cycle 1 and $500 \mathrm{mg} / \mathrm{m}^{2}$ iv cycles 2-6) every 28 days, for up to six cycles. Major exclusion criteria were prior treatment for CLL, severe cardiac, pulmonary, neurological, psychiatric, or metabolic disease, continuous systemic corticosteroids, active autoimmune hemolytic anemia or thrombocytopenia, active severe infection, creatinine clearance $<50 \mathrm{~mL} / \mathrm{min}$, or transformation to an aggressive B-cell malignancy. All cases were $\mathrm{CD} 20^{+}$as analyzed by flow cytometry, with a mean fluorescence intensity lower than the expression found in normal mature B lymphocytes in peripheral blood and bone marrow (BM).

At the 3-month post-induction clinical response evaluation, patients achieving a CR, partial response (PR) or nodular PR (nPR), based on International Workshop on CLL guidelines, were treated with rituximab $375 \mathrm{mg} / \mathrm{m}^{2}$ iv every 2 months for 3 years (18 cycles). Anti-microbial prophylaxis included trimethoprim-sulfamethoxazole and acyclovir during treatment and until the level of $\mathrm{CD} 4^{+}$lymphocytes reached $0.3 \times 10^{9} / \mathrm{L}$. Patients achieving a CR and undetectable MRD in both peripheral blood and BM after four courses of FCR were allowed to stop fludarabine plus cyclophosphamide and complete two courses of rituximab and continue with rituximab maintenance therapy.

The primary endpoint was the CR rate after FCR treatment. Secondary endpoints included PFS, OS, correlation of response with the level of MRD after FCR and rituximab maintenance therapy, adverse events, and the prognostic impact of the biological markers CD38 and ZAP70, IGHV mutational status, cytogenetic abnormalities and BM-MRD on the course of the disease. Fluorescence in situ hybridization and IGHV analysis were performed locally in accredited laboratories using standardized procedures.

The study protocol was approved by the institutional review board of each participating institution and complied with the Declaration of Helsinki, and existing Good Clinical Practice guidelines, laws and regulations. All participants provided written informed consent before enrollment.

Flow cytometry and measurable residual disease analysis

Samples were stained and lysed using a direct immunofluorescence technique as previously described. ${ }^{13}$ The following antibody combinations were used: (i) CD22/CD23/CD19/CD5; (ii) FMC7/CD43/CD19/CD5; (iii) CD103/CD25/CD19/CD5; (iv) CD10/CD11c/CD19/CD5; (v) CD20/CD38/CD19/CD5; (vi) CD81/CD22/CD19/CD5; (vii) CD20/CD49d/CD19/CD5; (viii) sIgk/sIgl/CD19/CD5, and (ix) ZAP70/CD3+CD56/CD19/CD5. All monoclonal antibodies except ZAP70 were provided by Becton Dickinson (San José, CA, USA). ZAP70 was purchased from Immunotech (Marseille, France). Samples were acquired in a FACSCalibur flow cytometer (Becton Dickinson) and analyzed using Paint-A-Gate PRO software (Becton Dickinson). At least 20,000 events were acquired. B-lymphocytes were identified according to their SSC/CD19+ distribution and the total percentage of pathological CD38 and CD49d B cells was reported. ZAP70 was quantified using a cut-off of $\geq 20 \%$ to define the $Z A P 70^{+}$subset of B cells. ${ }^{14}$

MRD was analyzed in samples from peripheral blood and BM after induction and from BM during rituximab maintenance therapy, with a combination of monoclonal antibodies slightly modified from that in the European Research Initiative on CLL (ERIC) protocol: (i) CD20/CD38/CD19/CD5;

CD81/CD22/CD19/CD5; (iii) sIgL/sIgK/CD19/CD5; and (iv) CD22/CD79b/CD19/CD5. CD43 was not included in the analysis: we included the last combination instead of CD43/CD79b/CD19/CD5 based on our previous experience with that combination in the analysis of MRD in CLL..$^{13}$ The minimum number of pathological $B$ cells acquired was that in the ERIC recommendations. ${ }^{15}$ To achieve a limit of detection of $0.01 \%$, at least 200,000 events were acquired if the minimum population size was 20 and 500,000 events if the minimum population size was 50 . We prepared the necessary number of tubes for each combination to acquire at least 200,000 events. The complete gating strategy is described in the Online Supplement.

\section{Statistical analysis}

This was a two-staged, Simon optimal phase II clinical trial. Based on a CR rate observed in previous trials of first-line therapy ranging around $30 \%$, the inactivity cut-off was chosen to equal $30 \%$ and the activity cut-off at least $50 \%$. Hence, the hypotheses of interest were $\mathrm{H}_{0}: r \leq 0.3$ against $\mathrm{H}_{\mathrm{a}}: r \geq 0.5 \%$, where $\mathrm{r}$ is the $\mathrm{CR}$ rate. Using a type I error rate ( $\alpha$, probability of accepting an insufficiently active treatment, a false positive outcome) set at 0.05 , and a type II error rate ( $\beta$, probability of rejecting an active treatment, a false negative outcome) set at 0.20 , we estimated that 90 patients should be enrolled into this trial, assuming a 10\% loss.

A descriptive analysis of continuous and qualitative variables was performed. PFS, OS and duration of response were summarized descriptively and graphically using the Kaplan and Meier method in the overall population and separately by biological factors, genetic profiles and MRD status. The log-rank test was used for comparisons of PFS and OS curves. The $\chi^{2}$ test was used to assess the frequencies and differences of biological and cytogenetic abnormalities. The relationship between these abnormalities and MRD level was analyzed using logistic regression models. Safety data were summarized for all treated patients during induction, maintenance and combined. All hypothesis tests were twosided and a $P$-value $<0.05$ was considered statistically significant. All statistical computations were carried out with SPSS version 14.0 or subsequent versions.

\section{Results}

We present the results of an end-of-study analysis at 3 years of follow-up after 36 months of rituximab maintenance therapy following FCR induction. Between October 2007 and December 2012, 90 patients were assessed for eligibility in 29 center across Spain, and 84 were assigned to FCR (6 patients did not meet the eligibility criteria, of whom 2 after 1 treatment infusion) and were evaluable for response in an intent-to-treat analysis (Figure 1). Overall, $79.8 \%(n=67)$ of the enrolled patients were aged 64 years or younger, $67.9 \%(n=57)$ were male and $83.3 \%(n=70)$ had Binet stage $\mathrm{B}$ or $\mathrm{C}$ disease. The median age of trial participants was 59.5 years (range, 37-70), and $70.5 \%(n=55)$ of participants were in a good state of health with an Eastern Cooperative Oncology Group Performance Status 
of $0-1$. Overall, $53.7 \%(n=45)$ of the trial population had B symptoms.

Table 1 summarizes the biological and genetic abnormalities assessed at baseline that were considered to be prognostic for outcome. Overall, $57.14 \% \quad(n=48)$ of patients had unmutated IGHV, 47.6\% $(n=39)$ were CD38 ${ }^{+}$ and $57.3 \% \quad(n=43)$ were ZAP70+. Forty-two $(50.0 \%)$ patients had a 13q14 deletion, $22(26.1 \%)$ had a 11q22-q23 deletion, $13(15.4 \%)$ harbored trisomy 12 , four $(4.7 \%)$ patients had a $17 \mathrm{p}$ deletion, and three $(3.5 \%)$ had a $6 \mathrm{q}$ deletion.

Overall, 12 patients (14\%) ended treatment induction prematurely. The reasons for discontinuation included toxicity $(n=6)$, progressive disease $(n=1)$, ineligibility $(n=2)$, and investigators' decision ( $n=3: 1$ patient with ischemic cerebrovascular disease, 1 patient with concomitant idiopathic thrombocytopenic purpura and 1 patient with a karyotype with chromosomal random losses). The median number of FCR cycles was six, and complete treatment was administered to $80 \%$ of the patients.

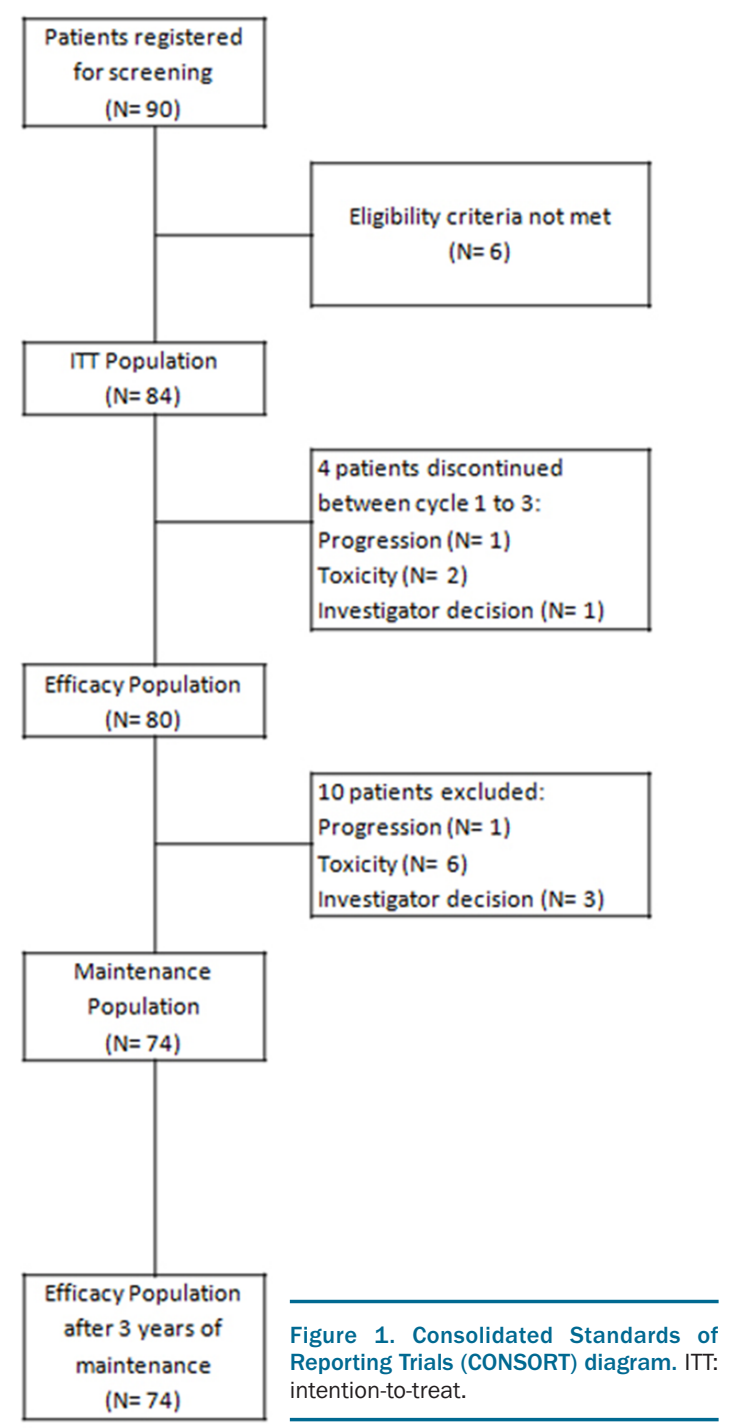

\section{Response and treatment outcomes} Induction

Of 84 evaluable patients in an intent-to-treat analysis of the effects of FCR induction treatment, 80 patients had a CR/CR with incomplete hematologic recovery (CRi), PR or $\mathrm{nPR}$ for an overall response rate of $95.2 \%(75.0 \%$ CR/CRi(2), $n=63 ; 7.1 \%$ nPR, $n=6 ; 13.1 \%$ PR, n=11) while four patients failed to respond to FCR. Of the 80 patients evaluable for BM-MRD status, $44.1 \%(n=37)$ had undetectable MRD at 3 months after induction, of whom 35 $(41.7 \%)$ had a CR and two $(2.4 \%)$ had a PR, while 43 had detectable MRD, of whom $28(35.0 \%)$ had a CR, eight $(10.0 \%)$ had a $\mathrm{nPR}$, and seven (8.8\%) had a PR.

\section{Rituximab maintenance}

Of the 80 patients with CR or PR after FCR induction, 74 entered the maintenance study. At the end of the maintenance phase, 52 patients had a CR and seven had a PR (2 nPR; 5 PR). At cycle 12 of treatment, 29 patients had a CR and four patients had attained a PR. At cycle 9, 42 patients had a CR and five had a PR. Reasons for discon-

Table 1. Patients' baseline characteristics.

\begin{tabular}{lc}
\hline Patients' characteristics & \\
Median age (range) & 59.5 years $(37,1-70,9)$ \\
Sex (n; \%) & \\
Men & $57(67.9)$ \\
Women & $27(32.1)$ \\
\hline ECOG performance status (n; \%) & \\
PS-0 & $55(70.5)$ \\
PS- $\geq 1$ & $23(29.4)$ \\
Binet stage (n; \%) & \\
A & $14(16.7)$ \\
B & $53(63.1)$ \\
C & $17(20.2)$ \\
\hline FISH cytogenetic status (n; \%) & \\
del(13q) & $42(50.0)$ \\
del(11q) & $22(26.1)$ \\
trisomy 12 & $13(15.4)$ \\
del(17p) & $4(4.7)$ \\
del(6q) & $3(3.5)$ \\
IGHV status (n; \%) & \\
unmutated & $48(57.1)$ \\
IGHV 3-21 & $3(3.6)$ \\
mutated & $27(32.1)$ \\
inconclusive & $6(7.1)$ \\
\hline Immunophenotyping (n; \%). & \\
>10\% CD38 positive & $53(64,6)$ \\
>30\% CD38 positive & $39(47,6)$ \\
>20\% ZAP70 positive & $43(57,3)$ \\
>20\% CD49d positive & $30(37,0)$ \\
Response (n; \%) & \\
Complete response & $63(75.0)$ \\
Partial response & $11(13.1)$ \\
Nodular partial response & $6(7.1)$ \\
\hline Undetectable MRD status post-induction & \\
Peripheral blood & $60(71.4)$ \\
Bone marrow & $37(44.0)$ \\
\hline ECOG: Easen Cooperise \\
\hline
\end{tabular}

ECOG: Eastern Cooperative Oncology Group; PS: Performance Status; FISH: fluorescence in-situ hybridization; MRD; measurable residual disease. 
tinuation were myelotoxicity ( $n=14 ; 18.9 \%)$, clinical progression ( $n=8 ; 10.8 \%)$, consent withdrawal ( $n=3 ; 4.0 \%)$ investigator's decision $(\mathrm{n}=1 ; 1.3 \%)$, protocol violation $(n=1 ; 1.3 \%)$, infection $(n=3 ; 4.0 \%)$ and death $(n=2 ; 2.7 \%)$. During the follow-up period, all patients who were analyzed maintained CR or PR.

At the end of maintenance therapy, MRD assessed at cycles 9, 12, 15 and 18 was negative in 44 of the 72 patients $(61.1 \%)$ evaluable for response. Interestingly, 29 patients who had detectable BM-MRD immediately after induction converted to an undetectable BM-MRD status following rituximab maintenance therapy. In detail, after nine cycles, 13 patients with detectable BM-MRD converted to having undetectable BM-MRD and two with undetectable BM-MRD became MRD-positive. After 18 cycles, 16 patients with detectable $\mathrm{BM}-\mathrm{MRD}$ converted to being MRD-negative and five with undetectable BMMRD became BM-MRD-positive (2 CR relapsed after 18 months, $1 \mathrm{CR}$ interrupted treatment at 12 months because of toxicity, 1 PR progressed after 13 months and $1 \mathrm{CR}$ relapsed after 4 months) (Table 2 ).

\section{Survival}

At the end of the study, with a median follow-up of 6.3 years, the estimated proportions of patients who were alive and progression-free were 0.76 and 0.61 , respectively. Analyzed according to their MRD status, patients with a $\mathrm{CR}$ and either undetectable or detectable MRD did not reach the median PFS and OS, while for patients with detectable MRD and a PR the median PFS was 2.04 years (95\% CI: $0-4.3$ years) and the median OS was 4.60 years (95\% CI: 3.0-6.1 years). Regarding response duration, a total of 56 patients $(71.2 \%)$ maintained their response throughout the whole study: the median response duration was 6.4 years (95\%CI: 6.08-6.68). Univariate Cox regression analysis showed that IGHV status affected PFS: the PFS rate at 7.3 years in patients with mutated IGHV was 0.85 , whereas it was 0.39 in those with unmutated IGHV. However, the median OS for patients with either mutated or unmutated IGHV was not reached (Figure 1). No correlations were identified between the other clinical, biological or molecular factors and the achievement of undetectable MRD.

When MRD values were categorized into low $(<0.01 \%$, i.e. less than 1 CLL cell per 10,000 leukocytes), intermediate $(0.01 \%$ to $1 \%)$ and high $(>1 \%)$, the median PFS and OS were not reached in patients with low and intermediate MRD levels and were 2.0 years (95\% CI: $0-4.3)$ and 4.6 years (95\% CI: 4.2-4.9), respectively, in patients with high MRD levels (Figure 2, and Table $3 \mathrm{~A}$ and $3 \mathrm{~B}$ ).

\section{Safety}

As per protocol, 86 patients were evaluated for safety after FCR induction. The most common adverse events were grade 1-2 rituximab infusion reactions (65.1\%), grade 3-4 myelosuppression (29 patients, 33.7\%) and infections (grade 1-2: 30 patients, $34.9 \%$; grade $3-4: 3$ patients, $3.5 \%)$. In addition, there were $11(12.8 \%)$ grade

Table 2. Measurable residual disease assessment.

\begin{tabular}{lcccc} 
MRD status & Induction $(n=74)$ & $\mathbf{6} \mathrm{Rm}$ cycles $(\mathbf{1}$ year) $(n=56)$ & $\mathbf{1 2}$ Rm cycles $(\mathbf{2}$ years) $(n=35)$ & 18 Rm cycles $(\mathbf{3}$ years) $(\mathbf{n = 4 2})$ \\
Negative & 33 & 36 & 23 & 31 \\
Positive & 41 & 20 & 12 & 11 \\
\hline
\end{tabular}

MRD: measurable residual disease; Rm: rituximab maintenance therapy
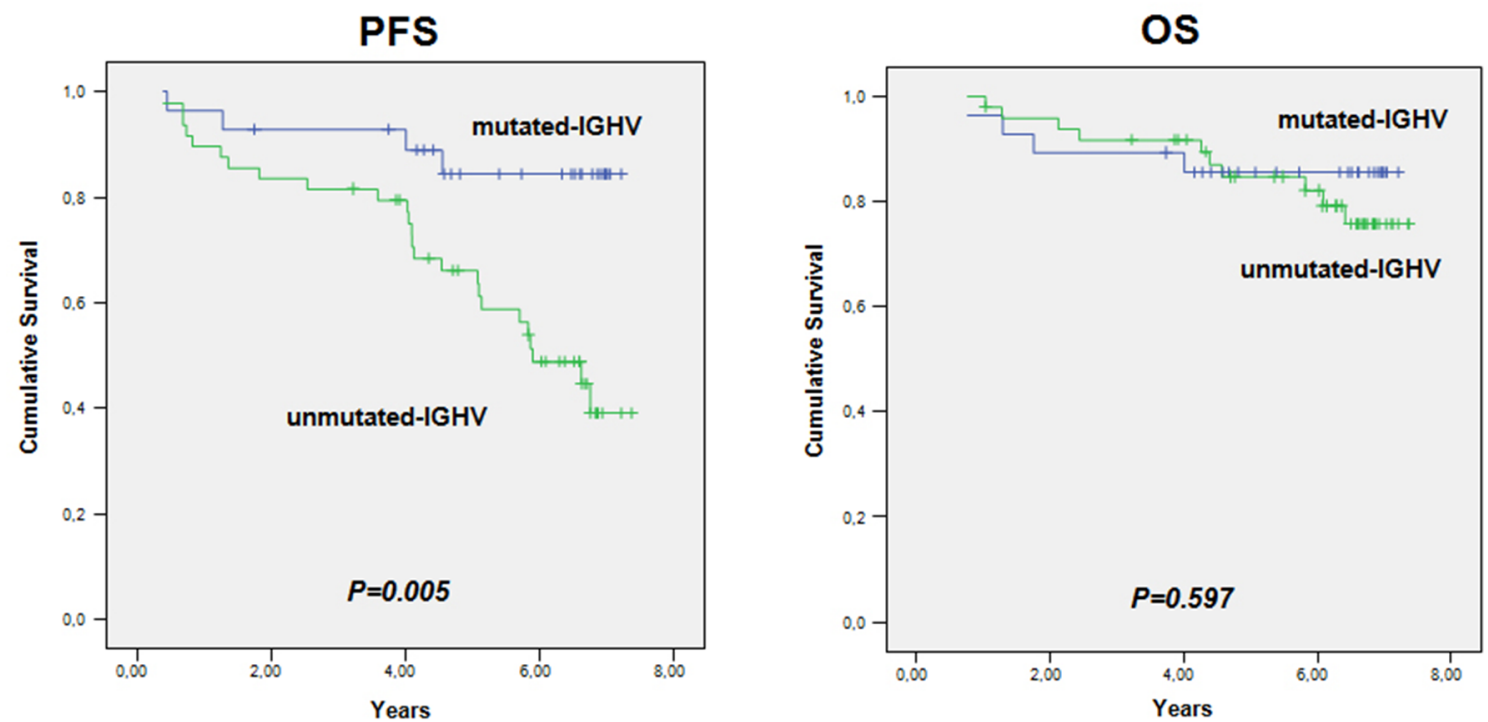

Figure 2. Progression-free survival and overall survival according to IGHV mutational status. PFS: progression-free survival; OS: overall survival. 
3-4 non-hematologic serious adverse events.

The most common adverse event during rituximab maintenance therapy was grade 3-4 myelosuppression, which occurred in 28 patients $(37.8 \%)$. In more detail, neutropenia between cycles and anemia were observed in 27 patients $(36.5 \%)$ and one patient $(1.4 \%)$, respectively. Grade 1-2 infections were detected in 43 patients $(58.1 \%)$, while grade 3-4 infections were documented in ten patients $(13.5 \%)$ and were pneumonia $(n=5)$, respiratory tract infections $(n=2)$, meningitis $(n=1)$, viral myocarditis $(n=1)$, and gastroenteritis $(n=1)$. Febrile neutropenia was observed in five patients $(6.8 \%)$.

\section{Discussion}

Despite the improved efficacy of currently approved chemoimmunotherapy in CLL patients, the majority of patients, including those who achieve CR, eventually relapse as a consequence of residual malignant cells still present after therapy. The high CR rate recorded in this study indicates that FCR induction followed by rituximab maintenance therapy produces a high overall response rate in patients considered fit for fludarabine-based therapy. By increasing the quality of clinical responses through obtaining a high undetectable MRD CR rate, the PFS of patients with a clinical response is prolonged. Ultimately, this confirms the role and value of undetectable MRD status in CLL.

In our study, MRD in BM was undetectable at the $10^{-4}$ level in $44 \%$ of the 80 patients evaluated after the induction treatment and in $68 \%$ of 59 patients at the end of maintenance therapy. Furthermore, rituximab maintenance therapy significantly increased the number of patients with undetectable MRD in BM. Indeed, 40 patients with detectable BM-MRD converted to an undetectable BM-MRD status from cycle 9 and subsequent

\section{PFS}

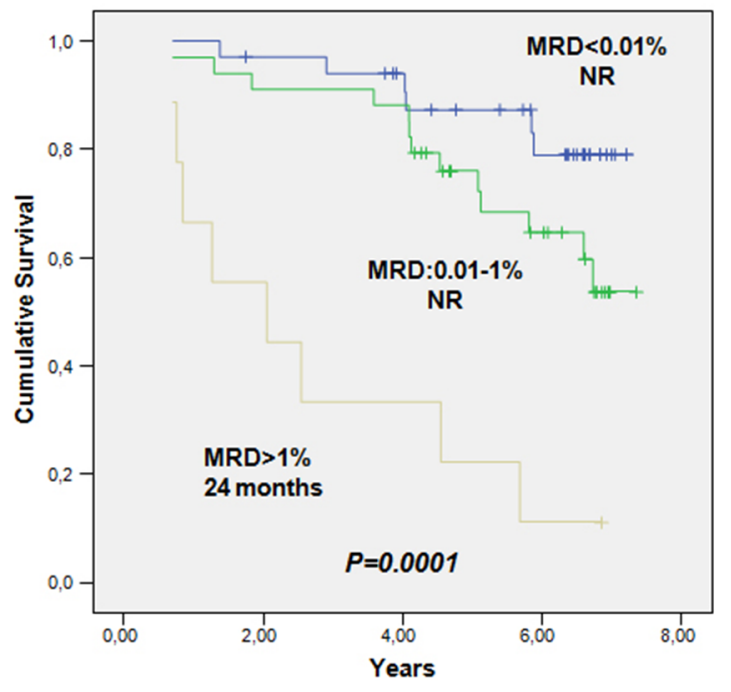

cycles. Additionally, although small numbers limited our subgroup analysis, it is remarkable that $41 \%, 58 \%$ and $60 \%$ of patients with undetectable MRD following rituximab maintenance therapy $(n=40)$ harbored factors wellknown to be associated with lower response and poor long-term outcomes. ${ }^{16-18}$ Our data suggest a PFS benefit from rituximab maintenance therapy in IGHV-mutated $v s$. unmutated patients (PFS at 7.2 year: $84.5 \%$ vs. $39.1 \%$, respectively). Overall, clinical outcomes were encouraging in this study as the median OS and PFS were not reached. The estimated 7-year PFS and OS rates were $56.2 \%$ and $78.0 \%$, respectively. Of note, the median OS and PFS for patients with a CR and either undetectable BM-MRD or detectable BM-MRD were not reached. However, despite these data suggesting a benefit on time-to-event curves, it is important to note that this was not a randomized study. Nevertheless, although direct comparisons between trials

Table 3A. Progression-free and overall survival according to measurable residual disease group assessment at the staging following treatment with fludarabine, cyclophosphamide, and rituximab.

\begin{tabular}{lccc} 
MRD Group & $\begin{array}{c}\text { Low } \\
(<0.01 \%)\end{array}$ & $\begin{array}{c}\text { Intermediate } \\
(\mathbf{0 . 0 1 \% - 1 \% )}\end{array}$ & $\begin{array}{c}\text { High } \\
(>1 \%)\end{array}$ \\
PFS & NR & NR & 2.0 years (95\% CI: $0-4.3)$ \\
OS & NR & NR & 4.6 years (95\% CI: $0-4.3)$ \\
\hline
\end{tabular}

MRD: measurable residual disease; PFS: progression-free survival; OS: overall survival; NR: not reached; $95 \% \mathrm{CI}$ : 95\% confidence interval.

Table 3B. Seven-year progression-free and overall survival rates after 36 months of maintenance therapy according to measurable residual disease group.

\begin{tabular}{lccc} 
MRD Group & $\begin{array}{c}\text { Low } \\
(<0.01 \%)\end{array}$ & $\begin{array}{c}\text { Intermedlate } \\
(\mathbf{0 . 0 1 \% - 1 \% )}\end{array}$ & $\begin{array}{c}\text { High } \\
(>1 \%)\end{array}$ \\
\hline PFS & $93.2 \%$ & $25.0 \%$ & $28.6 \%$ \\
OS & $100 \%$ & $100 \%$ & $68.6 \%$ \\
\hline
\end{tabular}

MRD: measurable residual disease; PFS: progression-free survival; OS: overall survival.

Figure 3. Progression-free survival and overall survival according to measurable residual disease status. PFS: progression-free survival; OS: overall survival; MRD: measurable residual disease; NR: not reached. 
is not recommended, these results (along with published data), suggest that maintenance treatment with a chemotherapy-free approach would improve long-term outcomes with acceptable toxicity. Furthermore, when $\mathrm{MRD}$ values were categorized into low $(<0.01 \%)$, intermediate $(0.01 \%$ to $1 \%)$ and high $(>1 \%)$, low and intermediate MRD levels were associated with longer PFS and OS following rituximab maintenance therapy (OS: $P<0.0001)$ compared with higher MRD levels which were associated with significantly shorter PFS and OS (2.0 and 4.6 years, respectively), suggesting a favorable prognostic effect of $M R D$ level for patients given rituximab maintenance therapy.

The relationship between undetectable MRD following frontline therapy and long-term outcomes, namely PFS and $O S$, has been investigated extensively in recent years. Nevertheless, to our knowledge, our study has one of the longest maintenance phases given to CLL patients in first remission. The German CLL Study Group (GCLLSG) updated the CLL8 trial which compared FCR to fludarabine plus cyclophosphamide in untreated CLL patients. With a median follow-up of 5.9 years, the median PFS for the patients treated with FCR was 56.8 months, and the median OS was not reached in the FCR group., Furthermore, patients achieving undetectable MRD had a significantly longer PFS (64.0 months) while the median $\mathrm{OS}$ was not reached. The rituximab-containing arm produced twice the number of patients achieving undetectable MRD.

In our study, median PFS and OS were not reached for patients with undetectable BM-MRD. We hypothesized that this difference was probably due to the source of samples. These data suggest that higher response rates and longer response durations could be expected by intensifying therapy through prolonged maintenance treatment with anti-CD20 immunotherapy. ${ }^{19}$ In addition, a combined analysis of the CLL8 and CLL10 trials showed that PFS was significantly longer in patients with undetectable $\mathrm{MRD}$ than in those with detectable $\mathrm{MRD}$, despite being unaffected by the residual tumor load, thus supporting the prognostic significance of undetectable MRD in CLL patients..$^{20}$ In another study Greil et al. enrolled patients who had achieved a CR, CRi or PR after first- or secondline rituximab-based chemoimmunotherapy. ${ }^{21}$ PFS was significantly longer in the rituximab maintenance arm (47.0 vs. 35.5 months, HR 0.50, 95\% CI: 0.38-0.66; $P<0.0001)$, suggesting that remission maintenance is an effective and safe option for CLL patients. In that study, $\mathrm{MRD}$ progression was documented more frequently in patients on observation than in those on rituximab maintenance therapy $(P<0.0001)$ and, interestingly, conversion to undetectable MRD status occurred more frequently in the rituximab maintenance arm (12 patients vs. 1 patient; $P=0.003)$. Based on these data, it seems that maintenance therapy may improve the quality of remission in CLL subjects and prolong PFS.

Although firm conclusions are limited by the number of subjects in our trial, patients who discontinued chemoimmunotherapy after achieving undetectable BM-MRD CR at cycle 4 and continued with the maintenance phase had similar PFS and OS rates to those of patients who achieved undetectable MRD but continued treatment for all six cycles: $93.3 \%$ and $76.5 \%$ were alive at 7 years, while $80.0 \%$ and $60.6 \%$ were free of disease at 7 years, respectively. In the light of these data, it might be useful to eval- uate the efficacy and efficiency of a strategy that adapts the duration of treatment to achieve undetectable MRD. Although a high rate of falsely negative MRD in peripheral blood up to 12 months has been reported with rituximab-containing regimens (20-30\%), our hypothesis was based on the predictive model published by Dimier et al., testing the effect of treatment on PFS using MRD as a surrogate endpoint. ${ }^{22}$ Thus, we only stopped FCR after four cycles when MRD was undetectable in both peripheral blood and BM. Although MRD-tailored therapy has not been validated prospectively, Strati et al. showed that patients who discontinued frontline FCR after achieving undetectable MRD after three cycles of treatment had similar PFS and OS to those who achieved undetectable MRD but continued treatment for all six cycles. ${ }^{23}$ Furthermore, PFS in the subgroup of patients who discontinued frontline FCR after three cycles of treatment because they had achieved undetectable MRD was better than the PFS of patients who remained with detectable $M R D$ at the end of the course of six cycles, despite the shorter duration of treatment in the former group. In addition, Thompson et al. recently reported that undetectable MRD after course 3 of FCR predicted a greater likelihood of undetectable MRD at the end of therapy. ${ }^{24}$

Although the study was designed before the ERIC recommended diagnostic markers were published, with a median number of BM leukocytes of around 410,000 (range, 150,000-610,000) and a sensitivity of MRD detection of $\geq 10^{-4}$, the immunophenotypic CLL analysis performed in this study was robust. The methodology for assessing MRD was similar to the flow cytometry methodology established by the ERIC consortium, as three of the tubes were similar to the subsequently published ERIC recommendations..$^{15,25,26}$ The potential limitation of the flow cytometry assay is the need for at least $10^{\circ}$ cells per tube and a total of four tubes, which may be an issue to keep in mind when small samples are available. In addition, selection of the sample source remains a challenge, as a significant discrepancy between MRD status determined in peripheral blood and $\mathrm{BM}$ has been reported. ${ }^{27}$ A paired analysis of peripheral blood and BM samples in our study revealed that 60 patients did not have detectable MRD in peripheral blood, while only 37 achieved undetectable MRD in the BM. This discrepancy is partially a result of the compartmental nature of CLL, with disease reservoirs in the BM, blood, lymph nodes, liver, and spleen. As rituximab targets CD20 on mature, malignant and benign $B$ cells, rituximab-based therapy will achieve undetectable MRD much more rapidly in peripheral blood than in BM. Indeed, in the REM trial we decided to use the CD20 marker in two of our MRD tubes for two reasons, (i) CD20 as a single marker provides the most powerful separation of CLL cells from normal B cells, and (ii) in patients treated with rituximab-containing regimens, the correlation between real-time quantitative polymerase chain reaction findings and the results of assays with combinations including the CD20 marker was not weaker than that with combinations not including the CD20 marker. ${ }^{28}$

Based on the results described above, it appears that 3 years of rituximab maintenance therapy was beneficial for enrolled patients, improving the quality of remissions and prolonging survival. The reason for the high response rates, undetectable MRD and favorable PFS and OS rates compared to those from clinical trials with similar entry 
criteria is probably multifactorial, but may include the age and Performance Status of the patients: their median age was 59.5 years and up to $70.5 \%$ patients had an Eastern Cooperative Oncology Group Performance Status of 0. Additionally, the protocol-defined anti-microbial prophylaxis scheduled for this trial allowed a treatment compliance of around $80 \%$. Furthermore, the secondary endpoint of undetectable MRD is strongly associated with outcome: at the post-maintenance assessment, $68 \%$ of assessed patients had undetectable MRD. Finally, the median follow-up of 75.6 months is long enough to allow solid interpretation of both PFS and OS.

A weakness of this trial is that, since its design, new drugs targeting signaling pathways, and newer monoclonal antibodies have become available, and the interest in chemoimmunotherapy, such as FCR, has weakened. Additionally, since OS findings have been inconsistent and one could argue that prolonged maintenance use of certain molecules could expose CLL patients to increased toxicity and ultimately reduce their quality of life, the debate should be whether to use rituximab for maintenance or to watch and wait and give these therapies when the patients relapse. Upon closer examination of our results, patients with unfavorable cytogenetics, unmutated somatic IGHV genes, and CD38 and ZAP70 expression benefited from rituximab maintenance therapy. Further research is now needed to identify subgroups of patients who may benefit while on maintenance therapy.

Of the 957 treatment-emergent adverse events, 54\% occurred during induction treatment; most were classed as neutropenia or lymphopenia, and almost half (47.4\%) were suspected to be related to rituximab. However, the same adverse events could be related to more than one of the drugs administered. The most frequent of the 440 treatment-emergent adverse events recorded during the maintenance period was neutropenia, which was recorded in $43.3 \%$ of the patients. Of the total of 957 treatmentemergent adverse events, $26.6 \%$ were assessed as grade $\geq 3$ and the majority were associated with disorders in the blood and lymphatic systems. Sixteen out of 20 deaths reported in the study occurred during the maintenance period. There was only one treatment-related death, which happened during the maintenance period. Overall, the safety profile of rituximab in the maintenance setting was consistent with its expected safety profile and no new unexpected adverse events were reported.

In summary, this study provides the first insights into the potential clinical use of FCR treatment followed by a 3 -year period of rituximab maintenance as a treatment strategy. Our study suggests that maintenance therapy with rituximab further prolongs responses in CLL patients with detectable MRD (when judged against historical outcomes after FCR treatment), with significantly improved PFS and OS for patients who achieved at least a PR after FCR induction. Based on these results, undetectable MRD is confirmed as a predictive biomarker associated with treatment response following rituximab maintenance therapy. Prospective studies aimed at evaluating long-term outcomes following early treatment discontinuation and the potential benefit in terms of reducing acute and delayed toxicity are necessary before $\mathrm{MRD}$ testing can be used to guide treatment decisions in clinical practice.

\section{Funding}

This work was funded by Roche Farma, S.A., Madrid, Spain.

\section{References}

1. Müller-Hermelink HK, Montserrat E, Catovsky D, et al. Chronic lymphocytic leukaemia/small lymphocytic lymphoma. In: Swerdlow SH, Campo E, Harris NL, et al., editors. WHO Classification of Tumours of Haematopoietic and Lymphoid Tissues. Lyon, France: IARC Press; 2008.pp.180-182.

2. Ginaldi L, De Martinis M, Matutes E, Farahat N, Morilla R, Catovsky D. Levels of expression of CD19 and CD20 in chronic B cell leukaemias. J Clin Pathol. 1998;51 (5):364-369.

3. Moreau EJ, Matutes E, A'Hern RP, et al. Improvement of the chronic lymphocytic leukemia scoring system with the monoclonal antibody SN8 (CD79b). Am J Clin Pathol. 1997;108(4):378-382.

4. Hallek M, Cheson BD, Catovsky D, et al. Guidelines for the diagnosis and treatment of chronic lymphocytic leukemia: a report from the International Workshop on Chronic Lymphocytic Leukemia updating the National Cancer Institute-Working Group 1996 guidelines. Blood. 2008;111 (12):5446-5456.

5. Hallek M, Fischer K, Fingerle-Rowson G, et al. Addition of rituximab to fludarabine and cyclophosphamide in patients with chronic lymphocytic leukaemia: a randomised, open-label, phase 3 trial. Lancet. 2010;376
(9747):1164-1174.

6. Rossi D, Terzi-di-Bergamo L, De Paoli L, et al. Molecular prediction of durable remission after first-line fludarabine-cyclophosphamide-rituximab in chronic lymphocytic leukemia. Blood. 2015;126(16):1921-1924.

7. Fischer K, Bahlo J, Fink AM, et al. Longterm remissions after FCR chemoimmunotherapy in previously untreated patients with CLL: updated results of the CLL8 trial. Blood. 2016;127(2):208-215.

8. Thompson PA, Tam CS, O'Brien SM, et al. Fludarabine, cyclophosphamide, and rituximab treatment achieves long-term diseasefree survival in IGHV-mutated chronic lymphocytic leukemia. Blood. 2016;127(3): 303-309.

9. Thompson PA, Wierda WG. Eliminating minimal residual disease as a therapeutic end point: working toward cure for patients with CLL. Blood. 2016;127(3):279-286.

10. Abrisqueta P, Villamor N, Terol MJ, et al. Rituximab maintenance after first-line therapy with rituximab, fludarabine, cyclophosphamide, and mitoxantrone (RFCM) for chronic lymphocytic leukemia. Blood. 2013;122(24):3951-3959.

11. Del Poeta G, Del Principe MI, Buccisano F, et al. Consolidation and maintenance immunotherapy with rituximab improve clinical outcome in patients with B-cell chronic lymphocytic leukemia. Cancer. 2008;112(1):119-128.

12. Bo MD, Del Principe MI, Pozzo F, et al.
NOTCH1 mutations identify a chronic lymphocytic leukemia patient subset with worse prognosis in the setting of a rituximab-based induction and consolidation treatment. Ann Hematol. 2014;93(10): 1765-1774

13. Garcia Vela JA, Delgado I, Benito L, et al. CD79b expression in B cell chronic lymphocytic leukemia: its implication for minimal residual disease detection. Leukemia. 1999;13(10):1501-1505.

14. Wang YH, Fan L, Xu W, Li JY. Detection methods of ZAP70 in chronic lymphocytic leukemia. Clin Exp Med. 2012;12(2):69-77.

15. Rawstron AC, Fazi C, Agathangelidis A. et al. A complementary role of multiparameter flow cytometry and high-throughput sequencing for minimal residual disease detection in chronic lymphocytic leukemia: an European Research Initiative on CLL study. Leukemia. 2016;30(4):929-936.

16. Hamblin TJ, Davis Z, Gardiner A, Oscier DG, Stevenson FK. Unmutated Ig V(H) genes are associated with a more aggressive form of chronic lymphocytic leukemia. Blood. 1999;94(6):1848-1854.

17. Hamblin TJ, Orchard JA, Ibbotson RE, et al CD38 expression and immunoglobulin variable region mutations are independent prognostic variables in chronic lymphocytic leukemia, but CD38 expression may vary during the course of the disease. Blood. 2002;99(3):1023-1029.

18. Dürig J, Nuckel H, Cremer M, et al. ZAP-70 
